\title{
Nonclassical Potential Symmetries of the Burgers Equation
}

\author{
Maria Luz GANDARIAS \\ Departamento de Matematicas, Universidad de Cadiz, \\ PO.BOX 40, 11510 Puerto Real, Cadiz, Spain \\ E-mail: mlgand@merlin.uca.es
}

\begin{abstract}
In this paper, new classes of symmetries for partial differential equations (PDE) which can be written in a conserved form are introduced. These new symmetries called nonclassical potential symmetries, are neither potential symmetries nor nonclassical symmetries. Some of these symmetries are carried out for the Burgers equation

$$
u_{t}+u u_{x}-u_{x x}=0 .
$$

by studying the nonclassical symmetries of the integrated equation

$$
v_{t}+\frac{v_{x}^{2}}{2}-v_{x x}=0 .
$$

By comparing the classical symmetries of the associated system

$$
\begin{aligned}
& v_{x}=u, \\
& v_{t}=u_{x}-\frac{u^{2}}{2}
\end{aligned}
$$

with those of the integrated equation (2), we deduce the condition for the symmetries of (2) to yield potential symmetries of (1). The nonclassical potential symmetries are realized as local nonclassical symmetries of (2). Similarity solutions are also discussed in terms of the integrated equation and yield solutions of the Burgers equation which are neither nonclassical solutions of the Burgers equation nor solutions arising from potential symmetries.
\end{abstract}

\section{Introduction}

Local symmetries admitted by a PDE are useful for finding invariant solutions. These solutions are obtained by using group invariants to reduce the number of independent variables.

The fundamental basis of the technique is that, when a differential equation is invariant under a Lie group of transformations, a reduction transformation exists. The machinery of the Lie group theory provides a systematic method to search for these special groupinvariant solutions. For PDE's with two independent variables, as it is equation (1), a single group reduction transforms the PDE into ODE's, which are generally easier to solve than the original PDE. Most of the required theory and description of the method can be found in $[6,12,13]$. 
Local symmetries admitted by a nonlinear PDE are also useful to discover whether or not the equation can be linearized by an invertible mapping and construct an explicit linearization when one exists. A nonlinear scalar PDE is linearizable by an invertible contact (point) transformation if and only if it admits an infinite-parameter Lie group of contact transformations satisfying specific criteria $[5,6,7,11]$.

An obvious limitation of group-theoretic methods based on local symmetries, in their utility for particular PDE's, is that many of these equations does not have local symmetries. It turns out that PDE's can admit nonlocal symmetries whose infinitesimal generators depend on the integrals of the dependent variables in some specific manner. It also happens that if a nonlinear scalar PDE does not admit an infinite-parameter Lie group of contact transformations, it is not linearizable by an invertible contact transformation. However, most of the interesting linearizations involve noninvertible transformations, such linearizations can be found by embedding given nonlinear PDE's in auxiliary systems of PDE's. [6].

Krasil'shchik and Vinogrod [15, 10] gave criteria which must be satisfied by nonlocal symmetries of a PDE when realized as local symmetries of a system of PDE's which 'covers' the given PDE. Akhatov, Gazizov and Ibragimov [1] gave nontrivial examples of nonlocal symmetries generated by heuristic procedures.

In $[5,6]$, Bluman introduced a method to find a new class of symmetries for a PDE. By writing a given $\mathrm{PDE}$, denoted by $\mathrm{R}\{\mathrm{x}, \mathrm{t}, \mathrm{u}\}$ in a conserved form, a related system denoted by $\mathrm{S}\{\mathrm{x}, \mathrm{t}, \mathrm{u}, \mathrm{v}\}$ with potentials as additional dependent variables is obtained. If $u(x, t), v(x, t)$ satisfies $\mathrm{S}\{\mathrm{x}, \mathrm{t}, \mathrm{u}, \mathrm{v}\}$, then $u(x, t)$ solves $\mathrm{R}\{\mathrm{x}, \mathrm{t}, \mathrm{u}\}$ and $v(x, t)$ solves an integrated related equation $\mathrm{T}\{\mathrm{x}, \mathrm{t}, \mathrm{v}\}$. Any Lie group of point transformations admitted by $\mathrm{S}\{\mathrm{x}, \mathrm{t}, \mathrm{u}, \mathrm{v}\}$ induces a symmetry for $\mathrm{R}\{\mathrm{x}, \mathrm{t}, \mathrm{u}\}$; when at least one of the generators of a group depends explicitly of on potential, then the corresponding symmetry is neither a point nor a Lie-Bäcklund symmetry. These symmetries of $\mathrm{R}\{\mathrm{x}, \mathrm{t}, \mathrm{u}\}$ are called potential symmetries.

The nature of potential symmetries allows one to extend the uses of point symmetries to such nonlocal symmetries. In particular:

1. Invariant solutions of $S\{x, t, u, v\}$, respectively $T\{x, t, v\}$, yield solutions of $R\{x, t, u\}$ which are not invariant solutions for any local symmetry admitted by $R\{x, t, u\}$.

2. If $R\{x, t, u\}$ admits a potential symmetry leading to the linearization of $S\{x, t, u, v\}$, respectively $\mathrm{T}\{\mathrm{x}, \mathrm{t}, \mathrm{v}\}$, then $\mathrm{R}\{\mathrm{x}, \mathrm{t}, \mathrm{u}\}$ is linearized by a noninvertible mapping.

Suppose $S\{x, t, u, v\}$ admits a local Lie group of transformations with the infinitesimal generator

$$
X_{S}=p(x, t, u, v) \frac{\partial}{\partial x}+q(x, t, u, v) \frac{\partial}{\partial t}+r(x, t, u, v) \frac{\partial}{\partial u}+s(x, t, u, v) \frac{\partial}{\partial v},
$$

this group maps any solution of $\mathrm{S}\{\mathrm{x}, \mathrm{t}, \mathrm{u}, \mathrm{v}\}$ to another solution of $\mathrm{S}\{\mathrm{x}, \mathrm{t}, \mathrm{u}, \mathrm{v}\}$ and hence induces a mapping of any solution of $\mathrm{R}\{\mathrm{x}, \mathrm{t}, \mathrm{u}\}$ to another solution of $\mathrm{R}\{\mathrm{x}, \mathrm{t}, \mathrm{u}\}$. Thus, (4) defines a symmetry group of $\mathrm{R}\{\mathrm{x}, \mathrm{t}, \mathrm{u}\}$. If

$$
\left(\frac{\partial p}{\partial v}\right)^{2}+\left(\frac{\partial q}{\partial v}\right)^{2}+\left(\frac{\partial r}{\partial v}\right)^{2} \neq 0
$$

then (4) yields a nonlocal symmetry of $\mathrm{R}\{\mathrm{x}, \mathrm{t}, \mathrm{u}\}$, such a nonlocal symmetry is called a potential symmetry of $\mathrm{R}\{\mathrm{x}, \mathrm{t}, \mathrm{u}\}$, otherwise $X_{S}$ projects onto a point symmetry of $\mathrm{R}\{\mathrm{x}, \mathrm{t}, \mathrm{u}\}$. 
Suppose

$$
X_{T}=p^{T}(x, t, v) \frac{\partial}{\partial x}+q^{T}(x, t, v) \frac{\partial}{\partial t}+s^{T}(x, t, v) \frac{\partial}{\partial v}
$$

defines a point symmetry of the related integrated equation $\mathrm{T}\{\mathrm{x}, \mathrm{t}, \mathrm{v}\}$. Then $X_{T}$ yields a nonlocal potential symmetry of $\mathrm{R}\{\mathrm{x}, \mathrm{t}, \mathrm{u}\}$ if and only if

$$
X_{S}=X_{T}+r(x, t, u, v) \frac{\partial}{\partial u}
$$

yields a nonlocal potential symmetry of $\mathrm{R}\{\mathrm{x}, \mathrm{t}, \mathrm{u}\}$.

Motivated by the fact that symmetry reductions for many PDE's are known that are not obtained using the classical Lie group method, there have been several generalizations of the classical Lie group method for symmetry reductions. Bluman and Cole developed the nonclassical method to study the symmetry reductions of the heat equation; Clarkson and Mansfield [8] presented an algorithm for calculating the determining equations associated with nonclassical symmetries.

The basic idea of the nonclassical method is that PDE is augmented with the invariance surface condition

$$
p u_{x}+q u_{t}-r=0
$$

which is associated with the vector field

$$
X_{R}=p(x, t, u) \frac{\partial}{\partial x}+q(x, t, u) \frac{\partial}{\partial t}+r(x, t, u) \frac{\partial}{\partial u} .
$$

By requiring that both (1) and (8) be invariant under the transformation with the infinitesimal generator (9), one obtains an overdetermined nonlinear system of equations for the infinitesimals $p(x, t, u), q(x, t, u), r(x, t, u)$. The number of determining equations arising in the nonclassical method is smaller than for the classical method, consequently the set of solutions is larger than for the classical method, as in this method one requires only the subset of solutions of (1) and (8) be invariant under the infinitesimal generator (9). However, associated vector fields do not form a vector space.

The determining equations, by applying the nonclassical method to the Burgers equation were first derived by Ames. This set of determining equations was partially solved by Pucci [14], Pucci also obtained, by using the nonclassical method due to Bluman, some new solutions of the Burgers equation which cannot be obtained by the direct method of Clarkson and Kruskal. In [2], Arrigo et al. formulate a criterion for determining when a solution obtained from a symmetry reduction of any equation calculated by Bluman and Cole's method is recoverable by the Clarkson and Kruskal approach and obtained some new solutions for the nonclassical determining equations of the Burgers equation as well as some new solutions for the Burgers equation.

Knowing that an associated system $\mathrm{S}\{\mathrm{x}, \mathrm{t}, \mathrm{u}, \mathrm{v}\}$ to the Boussinesq equation has the same classical symmetries as the Boussinesq equation, Clarkson proposed as an open problem if an auxiliary system $\mathrm{S}\{\mathrm{x}, \mathrm{t}, \mathrm{u}, \mathrm{v}\}$ of the Boussinesq equation does possess more or less nonclassical symmetries as compared with the equation itself.

Bluman says [3] that the ansatz to generate nonclassical solutions of $S\{x, t, u, v\}$ could yield solutions of $R\{x, t, u\}$ which are neither nonclassical solutions of $R\{x, t, u\}$ nor solutions arising from potential symmetries. However, as far as we know, none of these new symmetries have been obtained. 
The aim of this work is to obtain new symmetries that we will call nonclassical potential symmetries for the Burgers equation.

The basic idea is that the related integrated equation $\mathrm{T}\{\mathrm{x}, \mathrm{t}, \mathrm{v}\}$ is augmented with the invariance surface condition

$$
p v_{x}+q v_{t}-s=0
$$

which is associated with the vector field (6).

By requiring that both (1) and (10) be invariant under the transformation with infinitesimal generator (6), one obtains an overdetermined, nonlinear system of equations for the infinitesimals $p(x, t, v), q(x, t, v), s(x, t, v)$.

Then $X_{T}$ yields a nonclassical potential symmetry of $\mathrm{R}\{\mathrm{x}, \mathrm{t}, \mathrm{u}\}$ if and only if (7) yields a nonlocal symmetry of $\mathrm{R}\{\mathrm{x}, \mathrm{t}, \mathrm{u}\}$ which is not a classical potential symmetry.

This new symmetry is a potential symmetry of $R\{x, t, u\}$ which does not arise from a Lie symmetry of $T\{x, t, v\}$ but from a nonclassical symmetry of $T\{x, t, v\}$.

\section{Potential symmetries for the Burgers equation}

Let $\mathrm{R}\{\mathrm{x}, \mathrm{t}, \mathrm{u}\}$ be the Burgers equation (1)

$$
u_{t}+u u_{x}-u_{x x}=0 .
$$

In order to find the potential symmetries of (1), we write the equation in a conserved form

$$
u_{t}+\left(\frac{u^{2}}{2}-u_{x}\right)_{x}=0 .
$$

From this conserved form, the associated auxiliary system $S\{x, t, u, v\}$ is given by (3). If $(\mathrm{u}(\mathrm{x}), \mathrm{v}(\mathrm{x}))$ satisfies $(3)$, then $\mathrm{u}(\mathrm{x})$ solves the Burgers equation and $\mathrm{v}(\mathrm{x})$ solves the integrated Burgers equation (2).

If (6) is the infinitesimal generator that leaves (2) invariant, then (7) is the infinitesimal generator that leaves (3) invariant if and only if $p(x, t)=p^{T}(x, t), q(t)=q^{T}(t), s(x, t, v)=$ $s^{T}(x, t, v)$, and $r(x, t, u, v)=\left(s_{v}-p_{x}\right) u+s_{x}$.

Hence we obtain that $X_{T}$ yields a potential symmetry of (1) if and only if

$$
s_{v v} u+s_{x v} \neq 0 .
$$

Bluman [4] derived that (3) admits an infinite-parameter Lie group of point symmetries corresponding to the infinitesimal generator

$$
X_{s}=e^{\frac{v}{2}}\left[\left(\frac{s_{1}(x, t) u}{2}+\frac{\partial s_{1}(x, t)}{\partial x}\right) \frac{\partial}{\partial u}+s_{1} \frac{\partial}{\partial v}\right] .
$$

We can see (7) that (2) admits an infinite-parameter Lie group of point symmetries corresponding to the infinitesimal generator

$$
X^{T}=e^{\frac{v}{2}} s_{1}(x, t) \frac{\partial}{\partial v} .
$$

These infinite-parameter Lie groups of point symmetries yield a potential symmetry for (1). 


\section{Nonclassical symmetries of the integrated equation}

To obtain potential nonclassical symmetries of the Burgers equation, we apply the nonclassical method to the integrated equation (2). To apply the nonclassical method to (2), we require (2) and (10) to be invariant under the infinitesimal generator (6). In the case $q \neq 0$, without loss of generality, we may set $q(x, t, u)=1$. The nonclassical method applied to (2) gives rise to the following determining equations for the infinitesimals

$$
\begin{aligned}
& 2 \frac{\partial^{2} p}{\partial v^{2}}+\frac{\partial p}{\partial v}=0 \\
& -2 \frac{\partial^{2} s}{\partial v^{2}}+\frac{\partial s}{\partial v}+4 \frac{\partial^{2} p}{\partial v \partial x}-4 p \frac{\partial p}{\partial v}=0 \\
& \frac{\partial s}{\partial x}-2 \frac{\partial^{2} s}{\partial v \partial x}+2 \frac{\partial p}{\partial v} s+\frac{\partial^{2} p}{\partial x^{2}}-2 p \frac{\partial p}{\partial x}-\frac{\partial p}{\partial t}=0 \\
& 4 \frac{\partial^{2} p}{\partial v^{2}} \frac{\partial s}{\partial x}+2 \frac{\partial p}{\partial v} \frac{\partial s}{\partial x}-4 \frac{\partial^{4} s}{\partial v^{2} \partial x^{2}}+4 \frac{\partial p}{\partial v} \frac{\partial^{2} s}{\partial v \partial x}+4 \frac{\partial^{2} p}{\partial v \partial x} \frac{\partial s}{\partial v}+\frac{\partial s}{\partial t}+2 \frac{\partial p}{\partial x} s+ \\
& 4 \frac{\partial^{3} p}{\partial v^{2} \partial x} s+2 \frac{\partial^{2} p}{\partial v \partial x} s+\frac{\partial^{3} p}{\partial x^{3}}-4 \frac{\partial p}{\partial v} \frac{\partial^{2} p}{\partial x^{2}}-2 p \frac{\partial^{2} p}{\partial x^{2}}-2\left(\frac{\partial p}{\partial x}\right)^{2}- \\
& 8 \frac{\partial^{2} p}{\partial v \partial x} \frac{\partial p}{\partial x}+2 \frac{\partial^{4} p}{\partial v \partial x^{3}}-4 p \frac{\partial^{3} p}{\partial v \partial x^{2}}-\frac{\partial^{2} p}{\partial t \partial x}-2 \frac{\partial^{3} p}{\partial t \partial v \partial x}=0 .
\end{aligned}
$$

Solving these equations, we obtain

$$
\begin{aligned}
& p=\mathrm{p}_{1}(\mathrm{x}, \mathrm{t}) e^{-\frac{v}{2}}+\mathrm{p}_{2}(\mathrm{x}, \mathrm{t}), \\
& s=\left(s_{1} e^{\frac{v}{2}}+\left(2 p_{1} p_{2}-2 \frac{\partial p_{1}}{\partial x}\right) e^{-\frac{v}{2}}+\frac{2}{3} p_{1}^{2}\right) e^{-v}+s_{2},
\end{aligned}
$$

with $s_{1}=s_{1}(x, t)$ and $s_{2}=s_{2}(x, t)$. Substituting into the determining equations leads to

$$
p_{1}=0, \quad p=p_{2}(x, t), \quad s=\mathrm{s}_{1}(\mathrm{x}, \mathrm{t}) e^{-\frac{v}{2}}+\mathrm{s}_{2}(\mathrm{x}, \mathrm{t}),
$$

where $p_{2}, s_{1}$, and $s_{2}$ are related by

$$
\begin{aligned}
& \frac{\partial \mathrm{s}_{2}}{\partial x}+\frac{\partial^{2} \mathrm{p}_{2}}{\partial x^{2}}-2 \mathrm{p}_{2} \frac{\partial \mathrm{p}_{2}}{\partial x}-\frac{\partial \mathrm{p}_{2}}{\partial t}=0, \\
& -\frac{\partial^{2} \mathrm{~s}_{1}}{\partial x^{2}}+\frac{\partial \mathrm{s}_{1}}{\partial t}+2 \frac{\partial \mathrm{p}_{2}}{\partial x} \mathrm{~s}_{1}=0, \\
& \frac{\partial \mathrm{s}_{2}}{\partial t}+2 \frac{\partial \mathrm{p}_{2}}{\partial x} \mathrm{~s}_{2}+\frac{\partial^{3} \mathrm{p}_{2}}{\partial x^{3}}-2 \mathrm{p}_{2} \frac{\partial^{2} \mathrm{p}_{2}}{\partial x^{2}}-2 \frac{\partial \mathrm{p}_{2}}{\partial x}-\frac{\partial^{2} \mathrm{p}_{2}}{\partial t \partial x}=0 .
\end{aligned}
$$

It has been shown that (1) admits a potential symmetry when (11) is satisfied. As $s$ is given by (14), (11) is satisfied if and only if $s_{1} \neq 0$. If $s_{1}=0$, the symmetries obtained for (2) project on to point symmetries of (1). 
Although the previous equations are too complicated to be solved in general, some solutions can be obtained. Choosing $p_{2}=p_{2}(x), s_{1}=s_{1}(x)$ and $s_{2}=s_{2}(x)$, we can distinguish the following cases:

1. For $s_{2} \neq 0$ from (15), we obtain

$$
s_{2}=-\frac{\partial p_{2}}{\partial x}+p_{2}^{2}+k_{1}
$$

Substituting into (17), we have

$$
\frac{d^{3} \mathrm{p}_{2}}{d x^{3}}-2 \mathrm{p}_{2} \frac{d^{2} \mathrm{p}_{2}}{d x^{2}}+2 \mathrm{p}_{2}{ }^{2} \frac{d \mathrm{p}_{2}}{d x}+2 \mathrm{k}_{1} \frac{d \mathrm{p}_{2}}{d x}-4\left(\frac{d \mathrm{p}_{2}}{d x}\right)^{2}=0 .
$$

Multiplying by $p_{2}$ and integrating with respect to $x$, we have

$$
\mathrm{p}_{2} \frac{d^{2} \mathrm{p}_{2}}{d x^{2}}-2 \mathrm{p}_{2}{ }^{2} \frac{d \mathrm{p}_{2}}{d x}-\frac{1}{2}\left(\frac{\partial p_{2}}{\partial x}\right)^{2}+\frac{\mathrm{p}_{2}{ }^{4}}{2}+\mathrm{k}_{1} \mathrm{p}_{2}{ }^{2}+\mathrm{k}_{2}=0
$$

Dividing by $2 p_{2}^{2}$, setting $k_{1}=k_{2}=0$ and making

$$
p_{2}=-\frac{w^{2}}{\int w^{2}},
$$

(17) can be written as

$$
w^{\prime \prime}=0 \text {. }
$$

Consequently,

$$
w=a x+b \quad \text { and } \quad p_{2}=-\frac{a^{2} x^{2}+2 a b x+b^{2}}{\frac{a^{2} x^{3}}{3}+a b x^{2}+b^{2} x} .
$$

Substituting $p_{2}$ into (16), we obtain

$$
\begin{aligned}
s_{1}= & b_{0}\left(\frac{a^{5} x^{7} \log x}{45 b^{5}}-\frac{a^{4} x^{6} \log x}{45 b^{4}}+\frac{a^{2} x^{4} \log x}{15 b^{2}}+x^{2} \log x-\frac{13 a^{5} x^{7}}{1800 b^{5}}+\right. \\
& \left.\frac{4 a^{4} x^{6}}{525 b^{4}}-\frac{7 a^{2} x^{4}}{150 b^{2}}+\cdots\right)+a_{0}\left(\frac{a^{5} x^{7}}{45 b^{5}}-\frac{a^{4} x^{6}}{45 b^{4}}+\frac{a^{2} x^{4}}{15 b^{2}}+x^{2}+\cdots\right), \\
s_{2}= & \frac{2 a^{2} x+2 a b}{\frac{a^{2} x^{3}}{3}+a b x^{2}+b^{2} x} .
\end{aligned}
$$

Setting $b=0$,

$$
X=-\frac{3}{x} \partial_{x}+\partial_{t}+\left[e^{\frac{\log x+v}{2}}\left(i \mathrm{k}_{3} \sinh \left(\frac{5 \log x}{2}\right)+\mathrm{k}_{4} \cosh \left(\frac{5 \log x}{2}\right)\right)+\frac{6}{x^{2}}\right] \partial_{v} .
$$

2. For $s_{2}=0$, by solving (15), we obtain

$$
\begin{aligned}
& p_{2}=\sqrt{c_{1}} \tan \left(\sqrt{c_{1}}\left(x+c_{2}\right)\right) \quad \text { if } \quad c_{1}>0, \\
& p_{2}=-\frac{\sqrt{-c_{1}}\left[c_{2} \exp \left(2 \sqrt{-c_{1}} x\right)+1\right]}{c_{2} \exp \left(2 \sqrt{-c_{1}} x\right)-1} \text { if } c_{1}<0, \\
& p_{2}=-\frac{1}{x+c_{2}} \text { if } \quad c_{1}=0 .
\end{aligned}
$$


2.1. For $c_{1}>0$, setting $c_{1}=1, c_{2}=0$ and solving (16), we obtain $p_{2}=\tan (x), \quad s_{1}=k_{1} x \tan (x)+k_{2} \tan (x)+k_{1}$.

Solving the invariant surface condition, we obtain the nonclassical symmetry reduction

$$
z=t-\log (\sin (x)), \quad v=2 \log \left(-\frac{4}{2 \mathrm{k}_{1} \log \sin x+\mathrm{k}_{1} x^{2}+2 \mathrm{k}_{2} x+2 H(z)}\right) .
$$

Substitution of (19) into the integrated Burgers equation (2) leads to the ODE

$$
H^{\prime \prime}+H^{\prime}-k_{1}=0
$$

whose solution is

$$
H(z)=k_{4} e^{-z}+k_{1} z+k,
$$

from which we obtain that an exact solution for (2) is

$$
v=-2\left(\log \left(-2 \mathrm{k}_{4} \sin x-\mathrm{k}_{1} e^{t} x^{2}-2 \mathrm{k}_{2} e^{t} x-\left(2 \mathrm{k}_{1} t+2 k\right) e^{t}\right)-t-2 \log 2\right)
$$

and by (3) a new exact solution of the Burgers equation is

$$
u=-\frac{2\left(-2 \mathrm{k}_{4} \cos x-2 \mathrm{k}_{1} e^{t} x-2 \mathrm{k}_{2} e^{t}\right)}{-2 \mathrm{k}_{4} \sin x-\mathrm{k}_{1} e^{t} x^{2}-2 \mathrm{k}_{2} e^{t} x-\left(2 \mathrm{k}_{1} t+2 k\right) e^{t}} .
$$

2.2. For $c_{1}=0$, setting $c_{2}=0$,

$$
X=-\frac{1}{x} \partial_{x}+\partial_{t}+e^{\frac{v}{2}}\left(\mathrm{k}_{2} x^{2}-\frac{\mathrm{k}_{1}}{3 x}\right) \partial_{v}
$$

Solving the invariant surface condition leads to the similarity variable and to the implicit solution ansatz

$$
z=\frac{x^{2}}{2}+t, \quad H(z)=\frac{\frac{3 \mathrm{k}_{2} x^{4}}{4}-\mathrm{k}_{1} x}{3}+2 e^{-\frac{v}{2}} .
$$

Substituting into the integrated Burgers equation leads to the ODE

$$
H^{\prime \prime}-3 k_{2}=0,
$$

whose solution is

$$
H(z)=\frac{3 \mathrm{k}_{2} z^{2}}{2}+\mathrm{k}_{3} z+\mathrm{k}_{4}
$$

from which we obtain the exact solution

$$
v=2 \log \left(-\frac{48}{3 \mathrm{k}_{2} x^{4}+\left(36 \mathrm{k}_{2} t-12 \mathrm{k}_{3}\right) x^{2}+8 \mathrm{k}_{1} x+36 \mathrm{k}_{2} t^{2}-24 \mathrm{k}_{3} t+24 \mathrm{k}_{4}}\right)
$$

and by (3) a new exact solution of the Burgers equation is

$$
u=-\frac{2\left(12 \mathrm{k}_{2} x^{3}+2\left(36 \mathrm{k}_{2} t-12 \mathrm{k}_{3}\right) x+8 \mathrm{k}_{1}\right)}{3 \mathrm{k}_{2} x^{4}+\left(36 \mathrm{k}_{2} t-12 \mathrm{k}_{3}\right) x^{2}+8 \mathrm{k}_{1} x+36 \mathrm{k}_{2} t^{2}-24 \mathrm{k}_{3} t+24 \mathrm{k}_{4}} .
$$




\section{Concluding remarks}

In this work, we have introduced new classes of symmetries for the Burgers equation. If the Burgers equation is written in a conserved form, then a related system (3) and a related integrated equation (2) may be obtained. The ansatz to generate nonclassical solutions of the associated integrated equation (2) yields solutions of (1) which are neither nonclassical solutions of (1) nor solutions arising from potential symmetries.

\section{References}

[1] Akhatov I.Sh., Gazizov R.K. and Ibragimov N.H., J. Soviet. Math., 1991, V.55, 1401.

[2] Arrigo D.J., Broadbridge P. and Hill J.M., J. Math. Phys., 1993, V.34, 4692-4703.

[3] Bluman G.W., An overview of potential symmetries, in: Proceedings of AMS-SIAM, 1992.

[4] Bluman G.W., Potential symmetries and linearization, in: Proceedings of NATO Advanced Research Workshop, Kluwer, Exeter, 1992.

[5] Bluman G.W. and Kumei S., J. Math. Phys., 1980, V.21, 1019.

[6] Bluman G.W. and Kumei S., Symmetries and Differential Equations, Springer, Berlin, 1989.

[7] Bluman G.W. and Kumei S., Euro. J. Appl. Math., 1990, V.1, 189-216.

[8] Clarkson P.A. and Mansfield E.L., SIAM J. Appl. Math., 1994, V.55, 1693-1719.

[9] Gandarias M.L., J. Phys. A: Math. Gen., 1996, V.29, 5919-5934.

[10] Krasil'shchik I.S. and Vinogradov A.M., Acta Applic. Math., 1989, V.15, 161-209.

[11] Kumei S. and Bluman G.W., SIAM J. Appl. Math., 1982, V.42, 1157-1173.

[12] Olver P.J., Applications of Lie Groups to Differential Equations, Springer, Berlin, 1986.

[13] Ovsiannikov L.V., Group Analysis of Differential Equations, Academic Press, New York, 1982.

[14] Pucci E., J. Phys. A: Math. Gen., 1992, V.25, 2631-2640.

[15] Vinogradov A.M., Symmetries of Partial Differential Equations, Kluver, Dordrecht, 1989. 